\title{
Imaging in Appendicular Diverticulosis with Appendicitis
}

\author{
Aruna R. Patil' Bhushan Chaudhari' Satyajit Godhi \\ ${ }^{1}$ Department of Radiology, Apollo Hospitals, Bangalore, \\ Karnataka, India \\ ${ }^{2}$ Department of Gastrosurgery, Apollo Hospitals, \\ Bangalore, Karnataka, India \\ ${ }^{3}$ Department of Pathology, Apollo Hospitals, Bangalore, \\ Karnataka, India \\ J Gastrointestinal Abdominal Radiol ISGAR:2020;3(suppl S1):S85-S88
}

Swarna Shivakumar ${ }^{3}$

\begin{abstract}
Address for correspondence Aruna R. Patil, MD, DNB, FRCR, Department of Radiology, Apollo Hospitals, 154/11, Bannerghatta Road, Opposite I.I.M., Bangalore, Karnataka, 560076, India (e-mail: dr.arunarpatil@gmail.com).
\end{abstract}
Abstract
Keywords
- appendix
- diverticulosis
- computed tomography

Appendicular diverticulosis (AD) is an extremely rare condition. They are either incidentally detected in a normal or inflamed appendix or as diverticulitis manifesting clinically as appendicitis. It is commonly a radiological or pathological diagnosis. On computed tomography (CT), AD can mimic focal perforation. There are reported associations between $\mathrm{AD}$ and appendicular adenocarcinoma. This case reports the classical features of $A D$ on $C T$ with background appendicitis.

\section{Introduction}

Appendicular diverticulosis (AD) is an extremely rare condition. They are either incidentally detected in a normal or inflamed appendix or as diverticulitis manifesting clinically as appendicitis. It is commonly a radiological or pathological diagnosis. Computed tomography (CT) aids in the diagnosis of $\mathrm{AD}$, and the commonest differential is a focal perforation. There are reported associations between AD and appendicular adenocarcinoma. This case report highlights the classical features of $\mathrm{AD}$ on $\mathrm{CT}$ with background appendicitis.

\section{Case Report}

A 49-year-old male presented to the emergency department with right lower abdominal pain for 2 days, low-grade fever, and nausea. On clinical examination, the patient was febrile. Tenderness was elicited in the right iliac fossa, otherwise the abdomen was soft on palpation. Laboratory investigations revealed mild lymphocytosis. Contrast-enhanced CT (CECT) was ordered for further evaluation. Images were acquired in the venous phase after intravenous administration of Omnipaque (Iohexol $360 \mathrm{mg} / \mathrm{mL}$ ) at a dose of $1.5 \mathrm{~mL} / \mathrm{kg}$.

On CECT, the appendix was dilated and fluid-filled, measuring $12 \mathrm{~mm}$ in caliber. Diffuse wall thickening, enhancement, and periappendiceal fat stranding were seen. Additionally, multiple diverticular outpouchings were noted (at least eight to nine) from the appendix, which were not separately inflamed ( - Fig. 1A-C). No solid enhancing lesion was seen in the appendix. Cecum showed few diverticula ( - Fig. 1D). A diagnosis of acute appendicitis with incidental AD was made. The patient was taken up for laparoscopic appendectomy. Intraoperatively, the appendix was inflamed and hyperemic, with multiple nodules studded on the surface ( - Fig. 2A). Appendectomy was performed. The postoperative course was uneventful.

On pathological examination, the appendix showed multiple diverticular outpouchings (- Fig. 2B). On histopathology, the appendicular wall showed multiple outpouchings with inflammatory infiltrate. Areas of dysplasia were additionally noted. No obvious malignancy was seen ( - Fig. 3 A, B).

\section{Discussion}

$\mathrm{AD}$ is a rare condition occurring in $0.004(2.1 \%)$ of appendicectomies. ${ }^{1}$ It was first described by Kelynack in $1893 .{ }^{2}$ These are protrusions of the mucosa and submucosa through muscularis defect and hence are pseudodiverticula. There may be associated colonic diverticulosis. Some suggest that these occur due to raised intraluminal pressure secondary to
DOI https://doi.org/ $10.1055 / s-0040-1715538$ ISSN 2581-9933.
(C) 2020. Indian Society of Gastrointestinal and Abdominal Radiology. This is an open access article published by Thieme under the terms of the Creative Commons Attribution-NonDerivative-NonCommercial-License, permitting copying and reproduction so long as the original work is given appropriate credit. Contents may not be used for commercial purposes, or adapted, remixed, transformed or built upon. (https://creativecommons.org/licenses/by-nc-nd/4.0/)

Thieme Medical and Scientific Publishers Pvt. Ltd., A-12, 2nd Floor, Sector 2, Noida-201301 UP, India 



Fig. 1 (A, B) Coronal Contrast-enhanced computed tomography (CT) images show a dilated fluid-filled appendix with wall thickening and periappendicular inflammation (arrowhead). Arrows show multiple diverticular outpouchings from the appendicular lumen. (C) Note clear communication with the lumen. (D) Also noted is a cecal diverticulum.
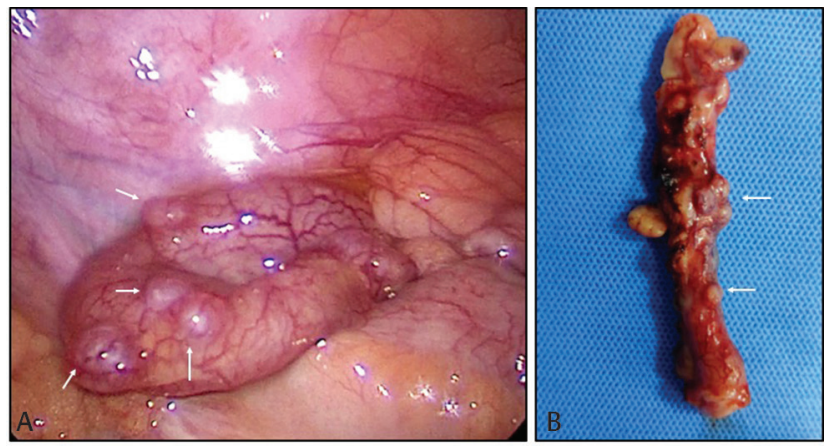

Fig. 2 (A) Intraoperative picture of appendicular diverticulosis seen as multifocal surface nodularity (arrows). Also note hyperemia suggesting inflammation. (B) Resected specimen reveals the same findings (arrows).

luminal obstruction by enterolith or inflammation or tumor. ${ }^{3}$ Imaging features of uncomplicated $\mathrm{AD}$ is rarely reported in the literature. They can be incidentally seen on barium enema studies conducted for different indications. ${ }^{4}$ The prevalence of appendicitis in AD is not clear as very few reports are available in the literature. ${ }^{5}$ Diverticulitis is, however, seen in two-thirds of cases..$^{6-8}$ Many of the diverticulitis cases were diagnosed based on postoperative histopathology rather than preoperative imaging, as differentiation from just appendicitis may be difficult on imaging unless the radiologists are aware of this entity and specifically look for them. Appendicular diverticulitis has a different epidemiology from

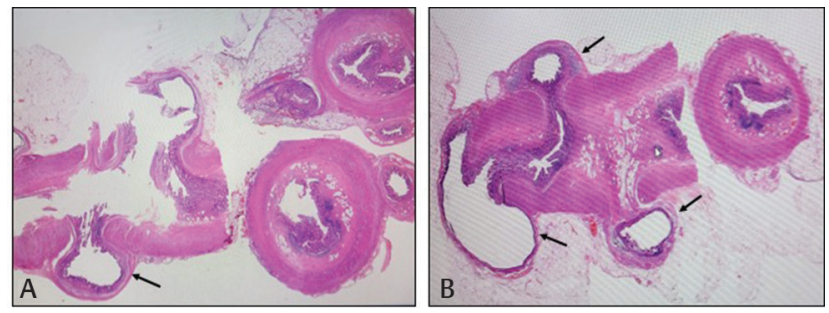

Fig. 3 (A, B) Low-magnification hematoxylin and eosin staining shows appendicular lumen with multiple diverticula (arrows) and areas of lymphocytic infiltration.



Fig. 4 Demonstration of the differences between appendicitis with contained perforation (A) and appendicular diverticulosis (B) on computed tomography.

pure appendicitis, with the latter being prevalent in older age group ${ }^{6,9}$ and presenting with intermittent pain. Early perforation, hemorrhage, and pseudomyxoma peritonei are reported with diverticulitis. ${ }^{6-8}$ On $\mathrm{CT}$, features are similar to those of diverticulosis elsewhere, for example, colonic. ${ }^{10}$ They can be fluid- or air-filled small outpouchings with a thin wall. Single outpouching in the appendiceal wall resembles focal perforation. Focal perforation, however, shows complete wall discontinuity with adjacent collection and significant fat inflammation ( - Fig. 4A, B). This differentiation is mandatory as contained perforations are initially managed conservatively including drainage followed by interval removal of the appendix. The association of AD with neoplasms has a high figure ( 7-48\%). ${ }^{11,12}$ Neoplasms reported include adenoma, adenocarcinoma, and carcinoids. Hence, isolated AD without inflammation is managed by prophylactic appendectomy. Though rare, due to eventual inflammation or tumor association, $\mathrm{AD}$ mandates diagnosis and mention in the radiology report whenever relevant features, as described, are seen.

\section{Conflict of Interest}

None declared.

\section{References}

1 Ng JL, Wong SL, Mathew R. Appendiceal diverticulosis: a harbinger of underlying primary appendiceal adenocarcinoma? J Gastrointest Oncol 2018;9(2):E1-E5

2 Kelynack TN, A Contribution to the Pathology of the Vermiform Appendix. London: HK Lewis; 1893:60-61

3 Abdullgaffar B. Diverticulosis and diverticulitis of the appendix. Int J Surg Pathol 2009;17(3):231-237

4 Mahmood RD. Appendiceal diverticulosis. BMJ Case Rep 2010;2010:bcr0720092090 
5 Käser SA, Willi N, Maurer CA. Prevalence and clinical implications of diverticulosis of the vermiform appendix. J Int Med Res 2013;41(4):1350-1356

6 Lock JH, Wheeler WE. Diverticular disease of the appendix. South Med J 1990;83(3):350

7 Kabiri H, Clarke LE, Tzarnas CD. Appendiceal diverticulitis. Am Surg 2006;72(3):221-223

8 Place RJ, Simmang CL, Huber PJ Jr. Appendiceal diverticulitis. South Med J 2000;93(1):76-79

9 Ito D, Miki K, Seiichiro S, et al. Clinical and computed tomography findings of appendiceal diverticulitis vs acute appendicitis. World J Gastroenterol 2015;21(13):3921-3927
10 Osada H, Ohno H, Saiga K, Watanabe W, Okada T, Honda N. Appendiceal diverticulitis: multidetector CT features. Jpn J Radiol 2012;30(3):242-248

11 Dupre MP, Jadavji I, Matshes E, Urbanski SJ. Diverticular disease of the vermiform appendix: a diagnostic clue to underlying appendiceal neoplasm. Hum Pathol 2008;39(12):1823-1826

12 Lamps LW, Gray GF Jr, Dilday BR, Washington MK. The coexistence of low-grade mucinous neoplasms of the appendix and appendiceal diverticula: a possible role in the pathogenesis of pseudomyxoma peritonei. Mod Pathol 2000;13(5):495-501 